Research Article

\title{
Generalized Type-I Hybrid Censoring Scheme in Estimation Competing Risks Chen Lifetime Populations
}

\author{
Neveen Sayed-Ahmed $\left(D^{1,2}\right.$ Taghreed M. Jawa, ${ }^{1}$ Tahani A. Aloafi, ${ }^{1}$ F. S. Bayones, ${ }^{1}$ \\ Azhari A. Elhag, ${ }^{1}$ J. Bouslimi, ${ }^{3}$ and G. A. Abd-Elmougod ${ }^{4}$ \\ ${ }^{1}$ Department of Mathematics and Statistics, College of Science, P.O. Box 11099, Taif University, Taif 21944, Saudi Arabia \\ ${ }^{2}$ Statistics Department, Faculty of Commerce (Girl Branch), Al-Azhar University, Cairo, Egypt \\ ${ }^{3}$ Department of Physics, College of Science, P.O. Box 11099, Taif University, Taif 21944, Saudi Arabia \\ ${ }^{4}$ Mathematics Department, Faculty of Science, Damanhour University, Damanhour, Egypt
}

Correspondence should be addressed to Neveen Sayed-Ahmed; nevensayd@yahoo.com

Received 16 November 2020; Revised 4 December 2020; Accepted 2 February 2021; Published 16 March 2021

Academic Editor: Antonio Mendes Lopes

Copyright (c) 2021 Neveen Sayed-Ahmed et al. This is an open access article distributed under the Creative Commons Attribution License, which permits unrestricted use, distribution, and reproduction in any medium, provided the original work is properly cited.

Different types of censoring scheme are investigated; however, statistical inference on censoring scheme which can save the ideal test time and the minimum number of failures is needed. The generalized type-I hybrid censoring scheme (GHCS) solves this problem. Competing the risk models under the GHCS when time to failure has Chen lifetime distribution (CD) is adopted in this research with consideration of only two cases of failure. Partially step-stress accelerated life tests (ALTs) are applied to obtain enough failure times in a small period to achieve a highly reliable product. The problem of parameter estimation under maximum likelihood (ML) and Bayes methods is discussed. The asymptotic confidence interval as well as the Bayes credible interval is constructed. The validity of theoretical results is assessed and compared through simulation study. Finally, brief comments are reported to describe the behaviour of the estimation results.

\section{Introduction}

Information about the lifetime products is presented in complete or censored data with respect to time or cost considerations. The complete failure time data is used when all the units under the test fail through the determined period of time. However, the censoring failure time data is used when some units under the test fail through a determined period of time. Various types of censoring are available and the common types are called type-I and type-II censoring schemes. The first scheme has a prefixed test time and a random number of failures but the second scheme has a prefixed number of failures and a random test time. In serval cases of censoring, the test is required to run joint case of type-I and type-II censoring schemes described by the hybrid censoring scheme (HCS). The HCS can be described statistically as follows: $\operatorname{suppose}\left(\tau, T_{m}\right)$ denote the ideal test time and the time of $m$ failure which is used for statistical inference, respectively, and the test is removed at the only one time of them. Then, HCS is defined under type-I and type-II censoring schemes and is called type-I HCS and type-II HCS. The test is removed at $\min \left(\tau, T_{m}\right)$ in the type-I HCS, but at $\max \left(\tau, T_{m}\right)$ in the type-II HCS, there is more information about the type of censoring presented by [1-3]. Furthermore, type-I censoring scheme or type-I HCS may satisfy the properties that the test has the smallest number of failures or maybe zero. However, type-II censoring scheme or type-II HCS satisfies the properties that the test has the largest number of failures; see [4]. The problem that appeared in these censoring schemes can be overcome in the generalized form of HSC; see [5] as type-I GHCS and type-II GHCS.

(1) In type-I GHCS, suppose $n$ independent units are put under test and the prior integers $r$ and $m$ satisfy that $1 \leq$ $r<m \leq n$ and prior time $\tau$. If the smallest number $r$ is satisfied before $\tau\left(T_{r}<\tau\right)$, then the test is terminated at $\min \left(T_{m}, \tau\right)$ and the observed test times data are given by 
$\underline{t}=\left(t_{1 ; n}<t_{1 ; n}<\cdots<t_{r ; n}<\cdots<t_{s ; n}\right), \quad r \leq s \leq m$.

However, if the smallest number $r$ does not satisfy before $\tau\left(\tau<T_{r}\right)$, then the test is terminated at $T_{r}$ and the observed test times data are given by

$$
\underline{t}=\left(t_{1 ; n}<t_{1 ; n}<\cdots<t_{r ; n}\right) .
$$

Finally, if the largest number $m$ is satisfied before $\tau$ $\left(T_{m}<\tau\right)$, then the test is terminated at $T_{m}$ and the observed test times are given by

$$
\underline{t}=\left(t_{1 ; n}<t_{1 ; n}<\cdots<t_{m ; n}\right) .
$$

Therefore, the type-I GHCS saves the minimum number which is necessary for statistical inferences.

(2) In type-II GHCS, let $n$ independent units be put under test and the two prior times $\tau_{1}$ and $\tau_{2}$ such that $\tau_{1}<\tau_{2}$ and integer $m$ satisfies $1 \leq m \leq n$. If the required number of failures is observed before $\tau_{1}$ $\left(T_{m}<\tau_{1}\right)$, then the test is terminated at $\tau_{1}$ and the observed test times data are given by

$$
\underline{t}=\left(t_{1 ; n}<t_{1 ; n}<\cdots<t_{m ; n}<\cdots<t_{s ; n}\right), \quad m \leq s \leq n .
$$

On the other hand, if the required number of failures observed satisfies $\tau_{1}<T_{m}<\tau_{2}$, then the test is terminated at $T_{m}$ and the observed test times data are given by

$$
\underline{t}=\left(t_{1 ; n}<t_{1 ; n}<\cdots<t_{m ; n}\right) .
$$

Finally, if the required number of failures is observed to satisfy $\tau_{1}<\tau_{2}<T_{m}$, then the test is terminated at $\tau_{2}$ and the observed test times are given by

$$
\underline{t}=\left(t_{1 ; n}<t_{1 ; n}<\cdots<t_{s ; n}\right), \quad 1 \leq s \leq m .
$$

In life testing experiments, the common problem is that units fail due to several fetal risks which are known as competing risks problem.

The effect of any risk factor in the presence of other risk factors need to be assessed. This problem has been discussed early in [6-10] and recently in [11]. Under the consideration of two causes of failure, the competing risks model in the presence of type-I GHCS is presented as follows.

For a randomly selected $n$ independent unit, a life testing experiment with priors integers $r$ and $m, 1 \leq r<m \leq n$, is considered. At each step of the experiment, time $T_{i ; n}$ and the cause of failure $\rho_{i}$ are recorded for $i=1,2, \ldots, d$, where $d$ satisfies $r<d<m$ and $\rho_{i} \in\{1,2\}$. Then, the joint likelihood function of type-I GHCS where $t=\left\{\left(t_{1 ; n}, \rho_{1}\right),\left(t_{2 ; n}, \rho_{2}\right), \ldots\right.$, $\left.\left(t_{d ; n}, \rho_{d}\right)\right\}$ under the competing risks model is reported as

$$
L(\underline{t} \mid \underline{\Theta})=A\left[S_{1}\left(t_{d}\right) S_{2}\left(t_{d}\right)\right]^{(n-d)} \prod_{i=1}^{d}\left[h_{1}\left(t_{i}\right)\right]^{\delta\left(\rho_{i}=1\right)}\left[h_{2}\left(t_{i}\right)\right]^{\delta\left(\rho_{i}=2\right)},
$$

where $A=n ! /(n-d) !, \quad S()=.1-F(),. \quad h()=.f(.) / F($.$) ,$ $\delta\left(\rho_{i}=j\right)=\left\{\begin{array}{ll}1, & \rho_{i}=j, \\ 0, & \rho_{i} \neq j,\end{array}\right.$ and for $t_{i}=t_{i ; n}, 0<t_{1}<t_{2}<\cdots<$ $t_{m}<\infty$.

To obtain more information about the lifetime of products industrial process, accelerated life tests (ALTs) present a suitable manner for reducing test time rather than using conditions. As we see in [12], ALTs are presented in different types; one of them is constant-stress ALTs, in which the test is kept with a constant level of stress; see [13-15]. The second type is called progressive-stress ALTs, in which the stress is kept with a continuously increasing level; see [16-18]. The third type is called step-stress ALTs, in which the stress level is changed through a prior time or the number of failures; see $[19,20]$. Furthermore, the ALTs can be done under the accelerated condition which is known by partial ALTs; see [21-26].

This paper aims to build and analyze type-I GHC competing risks sample under the model of partially stepstress ALTs from Chen lifetime products. The results of statistical analysis are built under maximum likelihood and Bayes method for point and interval estimation. The performances of the developed results are assessed and compared with mean squared error (MSE), average interval length $(\mathrm{AL})$, and probability coverage (PC) through the Monte Carlo study.

This paper is structured as follows: the model formulation and abbreviation are presented in Section 2. The MLEs of model parameters as well as the asymptotic confidence intervals are investigated in Section 3. Bayes estimation with credible intervals is discussed in Section 4 . The quality points and interval estimators are assessed via the Monte Carlo study in Section 5. Finally, the discussion and conclusion are presented in Section 6 (Table 1).

\section{Abbreviation and Model Formulation}

\subsection{Abbreviations}

2.1.1. Model Formulation. Suppose $n$ identical units are under life testing and two prior integer numbers $r$ and $m$ satisfy that $1 \leq r<m \leq n$ and prior fixed time $\tau$. The failure times $T_{i}$ and cause of failure $\rho_{i}$ are recorded through the test steps. If the smallest number of failure units $r$ is satisfied before time $\tau$, then the test is terminated at $\min \left(T_{m}, \tau\right)$. However, if $r$ is satisfied after the time $\tau$, then the test is terminated at $T_{r}$. The test is running under conditions until a fixed time $\eta$; then, the test is ruining under accelerated conditions. Considering that, the failure time has an independent $\mathrm{CD}$ and two independent causes of failure to satisfy the following assumptions:

(1) The random variable $T_{j i}$ is distributed with $\mathrm{CD}\left(\alpha, \beta_{j}\right), j=1,2$ with PDF given by

$$
f_{j 1}(t)=\alpha \beta_{j} t^{\alpha-1} e^{t^{\alpha}+\beta_{j}\left[1-e^{t^{\alpha}}\right]}, \quad t>0, \alpha, \beta_{j}>0,
$$

and $\mathrm{CDF}$ is given by 
TABLE 1: List of abbreviations.

\begin{tabular}{lc}
\hline PDF & Probability density function \\
CDF & Cumulative probability function \\
$t_{i}$ & The $i$-th unit failure time \\
$t_{j i}$ & The $i$-th unit failure time under cause $j, j=1,2$ \\
$f()$. & The PDF of $t_{i}$ \\
$F()$. & The CDF of $t_{i}$ \\
$S()$. & Survival function of $t_{i}$ \\
$h()$. & Hazard failure rate of $t_{i}$ \\
$f_{j}()$. & The PDF of $t_{j i}$ \\
$F_{j}()$. & The CDF of $t_{j i}$ \\
$S_{j}()$. & Survival function of $t_{j i}$ \\
$h_{j}()$. & Hazard failure rate function of $t_{j i}$ \\
$\rho_{i}$ & The cause of failure form $i$-th unit failure time \\
$\mathrm{CD}(\alpha, \beta)$ & Chen distribution with PDF $\alpha \beta t^{\alpha-1} e^{\left\{t^{\alpha}+\beta\left[1-e^{t^{\alpha}}\right]\right\}}$ \\
$\mathrm{MLE}$ & Maximum likelihood estimators \\
$\mathrm{AV}$ & Average estimate \\
$\mathrm{MSE}$ & Mean squared error \\
$\mathrm{AL}$ & Average interval length \\
$\mathrm{PC}$ & Probability coverage
\end{tabular}

$$
F_{j 1}(t)=1-e^{\beta_{j}\left[1-e^{t^{\alpha}}\right]}
$$

Also, $S_{j 1}(t)$ and $h_{j 1}(t)$ of $\mathrm{CD}\left(\alpha, \beta_{j}\right)$ are, respectively, given by

$$
\begin{aligned}
& S_{j 1}(t)=e^{\beta_{j}\left[1-e^{t^{\alpha}}\right]}, \\
& h_{j 1}(t)=\alpha \beta_{j} t^{\alpha-1} e^{t^{\alpha}} .
\end{aligned}
$$

(2) The random failure times $T_{i}, i=1,2, \ldots, d$, with the failure times under cause $j T_{j i}$, satisfy $T_{i}=\min \left\{T_{1 i}\right.$, $\left.T_{2 i}\right\}$.

(3) The total lifetime under use and accelerated condition $W$ under accelerated stress change $\eta$ is defined by

$$
W= \begin{cases}T, & T<\eta, \\ \eta+\frac{T-\eta}{\lambda}, & T>\eta,\end{cases}
$$

where $\lambda$ is the accelerated factor. The random variable $W$ is distributed with Chen lifetime distribution with PDF and is given by

$$
f_{l}(w)= \begin{cases}0, & w<0, \\ f_{j 1}(w), & 0<w<\eta, \\ f_{j 2}(w), & w \geq \eta,\end{cases}
$$

where

$f_{j 2}(w)=\alpha \lambda \beta_{j}(\eta+\lambda(w-\eta))^{\alpha-1} e^{(\eta+\lambda(w-\eta))^{\alpha}+\beta_{j}\left[1-e^{(\eta+\lambda(w-\eta))^{\alpha}}\right]}$, and $f_{j 1}(z)$ is given by (8). The CDF, $S_{j 2}(z)$, and hazard rate function $h_{j 2}(z)$ are given by

$$
\begin{aligned}
& F_{j 2}(w)=1-e^{\beta_{j}\left[1-e^{(\eta+\lambda(w-\eta))^{\alpha}}\right]}, \\
& S_{j 2}(w)=e^{\beta_{j}\left[1-e^{(\eta+\lambda(w-\eta))^{\alpha}}\right]}, \\
& h_{j 2}(w)=\alpha \lambda \beta_{j}(\eta+\lambda(w-\eta))^{\alpha-1} e^{(\eta+\lambda(w-\eta))^{\alpha}} .
\end{aligned}
$$

(4) Under competing risks type-I GHC sample and partially step-stress ALTs model, the test is terminated at $T_{r}$ at $\tau<T_{r}$ and $\min \left(\tau, T_{m}\right)$ at $\tau>T_{r}$. Then, the random sample of the total lifetime $W$ is described by

$$
\begin{aligned}
\underline{W} & =\left\{\left(W_{1}, \rho_{1}\right)<\left(W_{2}, \rho_{2}\right)<\cdots<\left(W_{k}, \rho_{k}\right)<\eta\right. \\
& \left.<\left(W_{k+1}, \rho_{k+1}\right)<\cdots<\left(W_{m}, \rho_{d}\right)\right\},
\end{aligned}
$$

where $d$ denotes the number of fail units, where $r<d<m$ and $k$ and $m-k$ are the numbers of fail units under using and accelerated conditions, respectively. For this model, we can consider three different cases, $\tau<\eta, \tau=\eta$, or $\tau<\eta$. Hence, the joint likelihood function of the observed values $\underline{w}=\left\{\left(w_{1}, \rho_{1}\right)<\left(w_{2}, \rho_{2}\right)<\cdots<\left(w_{k}, \rho_{k}\right)<\eta<\left(w_{k+1}, \rho_{k+1}\right)\right.$ $\left.<\cdots<\left(w_{m}, \rho_{d}\right)\right\}$ is obtained as follows:

$$
\begin{aligned}
L(\underline{t} \mid \underline{\Theta})= & A\left[S_{12}\left(w_{d}\right) S_{22}\left(w_{d}\right)\right]^{(n-d)} \prod_{i=1}^{k}\left[h_{11}\left(w_{i}\right)\right]^{\delta\left(\rho_{i}=1\right)} \\
& {\left[h_{21}\left(w_{i}\right)\right]^{\delta\left(\rho_{i}=2\right)} S_{11}\left(w_{i}\right) S_{21}\left(w_{i}\right) \times } \\
& \prod_{i=k+1}^{d}\left[h_{12}\left(w_{i}\right)\right]^{\delta\left(\rho_{i}=1\right)}\left[h_{22}\left(w_{i}\right)\right]^{\delta\left(\rho_{i}=2\right)} S_{12}\left(w_{i}\right) S_{22}\left(w_{i}\right) .
\end{aligned}
$$

\section{Maximum Likelihood Estimation}

When only two independent causes of failure and the test are running under the model of partially step-stress ALTs with type-I GHCS, the test information sample is used to obtain the point and interval MLEs which is reported in this section as follows.

3.1. MLEs. The joint likelihood function (18) under CDF (9) and (14) for the observed type-I GHC sample $\underline{w}=\left\{\left(w_{1}, \rho_{1}\right)\right.$ $\left.<\left(w_{2}, \rho_{2}\right)<\cdots<\left(w_{k}, \rho_{k}\right)<\eta<\left(w_{k+1}, \rho_{k+1}\right)<\cdots<\left(w_{m}, \rho_{d}\right)\right\}$ is given by 


$$
\begin{aligned}
& L\left(\alpha, \beta_{1}, \beta_{2}, \lambda \mid \underline{w}\right) \propto \alpha^{d} \beta_{1}^{m_{1}} \beta_{2}^{m_{2}} \lambda^{d-k} \prod_{i=1}^{k} w_{i}^{\alpha-1} \prod_{i=k+1}^{d}(\eta+\lambda(w-\eta))^{\alpha-1} \\
& e^{-\left(\beta_{1}+\beta_{2}\right)\left\{(n-d)\left[e^{\left(\eta+\lambda\left(w_{d}-\eta\right)\right)^{\alpha}}\right]+\sum_{i=1}^{k} e^{w_{i}^{\alpha}}+\sum_{i=k+1}^{d} e^{\left(\eta+\lambda\left(w_{i}-\eta\right)\right)^{\alpha}}-n\right\}} \\
& e^{\sum_{i=1}^{k} w_{i}^{\alpha}+\sum_{i=k+1}^{d}(\eta+\lambda(w-\eta))^{\alpha}},
\end{aligned}
$$

where integers $k$ and $(d-k)$ are denoted to failure units under using and stress conditions, respectively, and integers $m_{1}$ and $m_{2}$ denoted failure units under causes $\left(\rho_{1}, \rho_{2}\right)$. Then, the log-form from (19) is reduced to

$$
\begin{aligned}
\ell\left(\alpha, \beta_{1}, \beta_{2}, \lambda \mid \underline{w}\right)= & d \log \alpha+m_{1} \log \beta_{1}+m_{2} \log \beta_{2}+(d-k) \log \lambda-\left(\beta_{1}+\beta_{2}\right) \\
& \times\left\{(n-d) e^{\left(\eta+\lambda\left(w_{d}-\eta\right)\right)^{\alpha}}+\sum_{i=1}^{k} e^{w_{i}^{\alpha}}+\sum_{i=k+1}^{d} e^{\left(\eta+\lambda\left(w_{i}-\eta\right)\right)^{\alpha}}-n\right\} \\
& +(\alpha-1)\left\{\sum_{i=1}^{k} \log w_{i}+\sum_{i=k+1}^{d} \log [\eta+\lambda(w-\eta)]\right\}+\sum_{i=1}^{k} w_{i}^{\alpha} \\
& +\sum_{i=k+1}^{m}(\eta+\lambda(w-\eta))^{\alpha} .
\end{aligned}
$$

The partial derivatives of log-likelihood function (20) are reduced to the likelihood equations solved with some numerical methods to obtain the estimates as follows:

$$
0=\frac{\partial\left(\alpha, \beta_{1}, \beta_{2}, \lambda \mid \underline{w}\right)}{\partial \beta_{l}}, \quad l=1,2,
$$

is reduced to

$$
\begin{aligned}
& \beta_{1}=\frac{m_{1}}{(n-d) e^{\left(\eta+\lambda\left(w_{d}-\eta\right)\right)^{\alpha}}+\sum_{i=1}^{k} e^{w_{i}^{\alpha}}+\sum_{i=k+1}^{d} e^{\left(\eta+\lambda\left(w_{i}-\eta\right)\right)^{\alpha}}-n}, \\
& \beta_{2}=\frac{m_{2}}{(n-d) e^{\left(\eta+\lambda\left(w_{d}-\eta\right)\right)^{\alpha}}+\sum_{i=1}^{k} e^{w_{i}^{\alpha}}+\sum_{i=k+1}^{d} e^{\left(\eta+\lambda\left(w_{i}-\eta\right)\right)^{\alpha}}-n} .
\end{aligned}
$$

Also,

is reduced to

$$
0=\frac{\partial\left(\alpha, \beta_{1}, \beta_{2}, \lambda \mid \underline{w}\right)}{\partial \alpha}
$$

$$
\begin{aligned}
& \frac{d}{\alpha}-\left(\beta_{1}+\beta_{2}\right)\left\{(n-d)\left(\eta+\lambda\left(w_{d}-\eta\right)\right)^{\alpha} e^{\left(\eta+\lambda\left(w_{d}-\eta\right)\right)^{\alpha}} \log \left(\eta+\lambda\left(w_{d}-\eta\right)\right)\right. \\
& \left.\quad+\sum_{i=1}^{k} w_{i}^{\alpha} e^{w_{i}^{\alpha}} \log w_{i}+\sum_{i=k+1}^{d}\left(\eta+\lambda\left(w_{i}-\eta\right)\right)^{\alpha} e^{\left(\eta+\lambda\left(w_{i}-\eta\right)\right)^{\alpha}} \log \left(\eta+\lambda\left(w_{i}-\eta\right)\right)\right\} \\
& \quad+\sum_{i=1}^{k} \log w_{i}+\sum_{i=k+1}^{d} \log [\eta+\lambda(w-\eta)]+\sum_{i=1}^{k} w_{i}^{\alpha} \log w_{i} \\
& \quad+\sum_{i=k+1}^{m}(\eta+\lambda(w-\eta))^{\alpha} \log (\eta+\lambda(w-\eta))=0 \\
& 0=\frac{\partial\left(\alpha, \beta_{1}, \beta_{2}, \lambda \mid \underline{w}\right)}{\partial \lambda}
\end{aligned}
$$


which is reduced to

$$
\begin{aligned}
& \frac{(d-k)}{\lambda}-\left(\beta_{1}+\beta_{2}\right)\left\{\alpha(n-d)\left(w_{d}-\eta\right)\left(\eta+\lambda\left(w_{d}-\eta\right)\right)^{\alpha-1} e^{\left(\eta+\lambda\left(w_{d}-\eta\right)\right)^{\alpha}}\right. \\
& \left.\quad+\alpha \sum_{i=k+1}^{d}\left(w_{i}-\eta\right)\left(\eta+\lambda\left(w_{i}-\eta\right)\right)^{\alpha-1} e^{\left(\eta+\lambda\left(w_{i}-\eta\right)\right)^{\alpha}}\right\} \\
& \quad+(\alpha-1) \sum_{i=k+1}^{d} \frac{(w-\eta)}{\eta+\lambda(w-\eta)}+\alpha \sum_{i=k+1}^{m}\left(w_{i}-\eta\right)\left(\eta+\lambda\left(w_{i}-\eta\right)\right)^{\alpha-1}=0
\end{aligned}
$$

The likelihood equations are reduced to two nonlinear equations which are solved numerically with any iteration method such as Newton Raphson to obtain $\widehat{\alpha}$ and $\widehat{\lambda}$ which are used in (22) and (23) to present $\widehat{\beta}_{1}$ and $\widehat{\beta}_{2}$.

3.2. Interval Estimation. For the parameters vectors $\Theta=(\alpha$, $\left.\beta_{1}, \beta_{2}, \lambda\right)$, the second partial derivatives of (20) with respect to $\Theta_{i}$, where $\Theta_{1}=\alpha, \Theta_{2}=\beta_{1}, \Theta_{3}=\beta_{2}, \Theta_{4}=\lambda$, are given by

$$
\frac{\partial\left(\alpha, \beta_{1}, \beta_{2}, \lambda \mid \underline{w}\right)}{\partial \Theta_{i} \partial \Theta_{l}}, \quad i, l=1,2,3,4,
$$

and the Fisher information matrix $\Sigma$ is given by

$$
\Sigma=-E\left(\frac{\partial^{2}\left(\alpha, \beta_{1}, \beta_{2}, \lambda \mid \underline{w}\right)}{\partial \Theta_{i} \partial \Theta_{l}}\right)
$$

which is computed as the negative expectation of second partial derivatives (27). The approximate information matrix is used as the approximate form of the Fisher information matrix $\Sigma$ specially in a large sample. The approximate information matrix $\Sigma_{0}$ at the maximum likelihood estimates $\widehat{\Theta}=\left(\widehat{\alpha}, \widehat{\beta}_{1}, \widehat{\beta}_{2}, \widehat{\lambda}\right)$ is given by

$$
\Sigma_{0}=-\left(\frac{\partial^{2}\left(\alpha, \beta_{1}, \beta_{2}, \lambda \mid \underline{w}\right)}{\partial \Theta_{i} \partial \Theta_{l}}\right)_{\widehat{\Theta}=\left\{\widehat{\alpha}, \widehat{\beta}_{1}, \widehat{\beta}_{2}, \hat{\lambda}\right\}} .
$$

The asymptotic normality distribution of estimating $\widehat{\alpha}$, $\widehat{\beta}_{1}, \widehat{\beta}_{2}$, and $\hat{\lambda}$ with mean $\left(\alpha, \beta_{1}, \beta_{2}, \lambda\right)$ and a variance covariance matrix $\Sigma_{0}^{-1}\left(\widehat{\alpha}, \widehat{\beta}_{1}, \widehat{\beta}_{2}, \widehat{\lambda}\right)$ is as follows:

$$
\left(\widehat{\alpha}, \widehat{\beta}_{1}, \widehat{\beta}_{2}, \widehat{\lambda}\right) \text { distributed as } N\left(\left(\alpha, \beta_{1}, \beta_{2}, \lambda\right), \Sigma_{0}^{-1}\left(\widehat{\alpha}, \widehat{\beta}_{1}, \widehat{\beta}_{2}, \widehat{\lambda}\right)\right) \text {. }
$$

Therefore, $100(1-\xi)$ intervals estimation of parameters vector $\Theta=\left\{\alpha, \beta_{1}, \beta_{2}, \lambda\right\}$ are computed by

$$
\widehat{\Theta}_{i} \mp z_{\xi / 2} \sqrt{\Sigma_{i i}}, \quad i=1,2,3,4,
$$

where $\widehat{\Theta}_{i}$ denotes the parameters estimate and value $\Sigma_{i i}$ denotes the diagonal of variance covariance matrix $\Sigma_{0}^{-1}$ with standard normal probability $\xi / 2$.

\section{Bayesian Approach with MCMC}

Information about the model parameters and the information which is obtained from the life sample is used in this section to build the Bayes approach with the MCMC method. Besides, the estimators of parameters of $\mathrm{CD}$ and noninformative about accelerated factor are computed under squared error loss (SEL) function and independent prior distributions. Therefore, independent gamma prior is adapted as follows:

$$
\pi_{l}^{*}\left(\Theta_{l}\right) \propto \Theta_{l}^{a_{l}-1} e^{-b_{l} \Theta_{l}}, \quad l=1,2,3, \text { and } \pi_{4}^{*}\left(\Theta_{4}\right) \propto \Theta_{4}^{-1},
$$

$$
\pi^{*}(\underline{\Theta})=\prod_{l=1}^{4} \pi_{l}^{*}\left(\Theta_{l}\right),
$$

where $\Theta=\left(\alpha, \beta_{1}, \beta_{2}, \lambda\right)$. Then, the posterior distribution of $\Theta$ is defined by

$$
\pi(\underline{\Theta} \mid \underline{w})=\frac{\pi^{*}(\underline{\Theta}) L(\underline{\Theta} \mid \underline{w})}{\int_{\underline{\Theta}} \pi^{*}(\underline{\Theta}) L(\underline{\Theta} \mid \underline{w}) \mathrm{d} \underline{\Theta}} .
$$

Then, the Bayes estimate for any function $\phi(\underline{\Theta})$ under SEL function is given by

$$
\widehat{\phi}_{B}(\underline{\Theta})=\frac{\int_{\underline{\Theta}} \phi(\underline{\Theta}) \pi^{*}(\underline{\Theta}) L(\underline{\Theta} \mid \underline{w}) \mathrm{d}(\underline{\Theta})}{\int_{(\underline{\Theta})} \pi^{*}(\underline{\Theta}) L(\underline{\Theta} \mid \underline{w}) \mathrm{d}(\underline{\Theta})} .
$$

Generally, the ratio in (35) needs numerical approximation to compute, such as numerical integration and Lindley approximation. However, MCMC methods are the important tools that were applied recently with high accuracy and are obtained as follows.

4.1. Gibbs with $M H$ Method. The posterior distribution in (34) with prior distribution (33) and likelihood function (19) is calculated as; 


$$
\begin{aligned}
& \pi\left(\alpha, \beta_{1}, \beta_{2}, \lambda \mid \underline{w}\right) \propto \alpha^{a_{1}+d-1} \beta_{1}^{a_{2}+m_{1}-1} \beta_{2}^{a_{3}+m_{2}-1} \lambda^{d-k-1} \prod_{i=1}^{k} w_{i}^{\alpha-1} \prod_{i=k+1}^{d}(\eta+\lambda(w-\eta))^{\alpha-1} \\
& \cdot e^{-\left(\beta_{1}+\beta_{2}\right)\left\{(n-d)\left[e^{\left(\eta+\lambda\left(w_{d}-\eta\right)\right)^{\alpha}}\right]+\sum_{i=1}^{k} e^{w_{i}^{\alpha}}+\sum_{i=k+1}^{d} e^{\left.\left(\eta+\lambda\left(w_{i}-\eta\right)\right)^{\alpha}-n\right\}}\right.} \\
& \cdot e^{-b_{1} \alpha-b_{2} \beta_{1}-b_{3} \beta_{2}+\sum_{i=1}^{k} e^{w_{i}^{\alpha}}+\sum_{i=k+1}^{m}(\eta+\lambda(w-\eta))^{\alpha}} .
\end{aligned}
$$

Then, the conditional PDFs of the posterior distribution is given by

$$
\begin{aligned}
& \pi_{1}\left(\alpha\left|\beta_{1}, \beta_{2}, \lambda\right| \underline{w}\right)=\alpha^{a_{1}+d-1} e^{-\left(\beta_{1}+\beta_{2}\right)}\left\{(n-d)\left[e^{\left(\eta+\lambda\left(w_{d}-\eta\right)\right)^{\alpha}}\right]+\sum_{i=1}^{k} e^{w_{i}^{\alpha}}+\sum_{i=k+1}^{d} e^{\left(\eta+\lambda\left(w_{i}-\eta\right)\right)^{\alpha}}\right\} \\
& e^{-b_{1} \alpha+\sum_{i=1}^{k} w_{i}^{\alpha}+\sum_{i=k+1}^{m}(\eta+\lambda(w-\eta))^{\alpha}} \prod_{i=1}^{k} w_{i}^{\alpha-1} \prod_{i=k+1}^{d}(\eta+\lambda(w-\eta))^{\alpha-1}, \\
& \pi_{2}\left(\beta_{1}\left|\alpha, \beta_{2}, \lambda\right| \underline{w}\right) \propto \beta_{1}^{a_{2}+m_{1}-1} e^{-\beta_{1}\left\{b_{2}+(n-d)\left[e^{\left(\eta+\lambda\left(w_{d}-\eta\right)\right)^{\alpha}}\right]+\sum_{i=1}^{k} e^{w_{i}^{\alpha}}+\sum_{i=k+1}^{d} e^{\left(\eta+\lambda\left(w_{i}-\eta\right)\right)^{\alpha}}-n\right\}},
\end{aligned}
$$

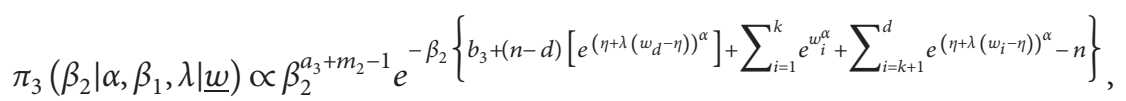

$$
\begin{aligned}
& \pi_{4}\left(\lambda \mid \alpha, \beta_{1}, \beta_{2}, \underline{w}\right) \propto \lambda^{d-k-1} e^{-\left(\beta_{1}+\beta_{2}\right)}\left[(n-d)\left[e^{\left(\eta+\lambda\left(w_{d}-\eta\right)\right)^{\alpha}}\right]+\sum_{i=k+1}^{k} e^{\left(\eta+\lambda\left(w_{i}-\eta\right)\right)^{\alpha}}\right] \\
& +\sum_{i=k+1}^{m}(\eta+\lambda(w-\eta))^{\alpha} \\
& \prod_{i=k+1}^{d}(\eta+\lambda(w-\eta))^{\alpha-1} .
\end{aligned}
$$

From equations (37) and (38), the conditional posterior PDFs are reduced to two conditional gamma density equations (38) and (39). Two functions are plotted similar to the normal distribution in (37) and (40). Then, the process of generation from posterior distribution under the conditional posterior distribution by using Gibbs with the $\mathrm{MH}$ algorithms with normal proposal distribution [27] is given as follows:

(1) Begin with initial vectors $\Theta^{(0)}=\left(\alpha^{(0)}, \beta_{1}^{(0)}, \beta_{2}^{(0)}, \lambda^{(0)}\right)$ and indicator $\kappa=1$.

(2) The two values $\beta_{1}^{(\kappa)}$ and $\beta_{2}^{(\kappa)}$ are generated from conditional gamma densities (38) and (39).

(3) The two values $\alpha^{(\kappa)}$ and $\lambda^{(\kappa)}$ are generated from conditional densities (37) and (40) by MH algorithms with normal proposal distributions. The symmetric normal distributions are applied with mean $\alpha^{(\kappa-1)}$ or $\lambda^{(\kappa-)}$ and variance obtained from the diagonal of the approximate information matrix, respectively. Also, the generated values are accepted with acceptance probability $\min \left[1,\left(\pi_{4}\left(\alpha^{(\kappa)}\right)\right.\right.$ $\left.\left.\left.\lambda^{(\kappa-1)}, \beta_{1}^{(\kappa)}, \beta_{2}^{(\kappa)}, \underline{w}\right) / \pi_{4}\left(\alpha^{(\kappa-1)} \mid \quad \lambda^{(\kappa-1)}, \beta_{1}^{(\kappa)}, \beta_{2}^{(\kappa)}, \underline{w}\right)\right)\right]$ or $\min \left[1,\left(\pi_{4}\left(\lambda^{(\kappa)} \mid \alpha^{(\kappa)}, \beta_{1}^{(\kappa)}, \beta_{2}^{(\kappa)}, \underline{w}\right) / \pi_{4}\left(\lambda^{(\kappa-1)} \mid \alpha^{(\kappa)}\right.\right.\right.$, $\left.\left.\left.\beta_{1}^{(\kappa)}, \beta_{2}^{(\kappa)}, \underline{w}\right)\right)\right]$, respectively, with respect to uniform $(0,1)$.

(4) The vector $\Theta^{(\kappa)}=\left(\alpha^{(\kappa)}, \beta_{1}^{(\kappa)}, \beta_{2}^{(\kappa)}, \lambda^{(\kappa)}\right)$ is a built vector with Gibbs manner.

(5) Put $\kappa=1+1$ and then repeat steps 2-4 $N$ times.

(6) The Bayes estimates and the corresponding variance are given by $\widehat{\Theta}_{B}=(1 / N-N) \sum_{i=N+1}^{N} \Theta^{(i)}$ and $\operatorname{Var}\left(\widehat{\Theta}_{B}\right)=(1 / N-N) \quad \sum_{i=N+1}^{N} \quad\left(\widehat{\Theta}_{B}-\Theta^{(i)}\right)^{2}$, where $\mathbf{N}$ is the number of iteration used to get stationary distribution.

(7) The Bayes credible intervals can be obtained after ordered values of $\Theta_{l}^{(\kappa)}$ in acceding order of $\Theta_{l}^{(\mathbf{N}+1)}$, $\Theta_{l}^{(\mathrm{N}+2)}, \ldots, \Theta_{l}^{[N]}$ as $\Theta_{l}^{[1]}, \Theta_{l}^{[2]}, \ldots, \Theta_{l}^{[N-\mathrm{N}]}$. Then, the $100(1-\zeta) \%$ symmetric credible intervals are obtained as $\left(\Theta_{l}^{(N-N)(\zeta / 2)}, \Theta_{l}^{(N-N)(1-(\zeta / 2))}\right), \quad l=$ $1,2,3,4$. 
TABle 2: The AV and MSE of parameters estimates for $\Theta(2,0.1,0.2,2.0)$.

\begin{tabular}{|c|c|c|c|c|c|c|c|c|c|c|c|c|c|}
\hline \multirow{2}{*}{$(\eta, \tau)$} & \multirow{2}{*}{$(r, m, n)$} & \multicolumn{4}{|c|}{ MLE } & \multicolumn{4}{|c|}{ MCMC/Prior 0} & \multicolumn{4}{|c|}{ MCMC/Prior 1} \\
\hline & & $\alpha$ & $\beta_{1}$ & $\beta_{1}$ & $\lambda$ & $\alpha$ & $\beta_{1}$ & $\beta_{1}$ & $\lambda$ & $\alpha$ & $\beta_{1}$ & $\beta_{1}$ & $\lambda$ \\
\hline \multirow{12}{*}{$(0.8,1.0)$} & \multirow{2}{*}{$(15,20,30)$} & 2.321 & 0.152 & 0.231 & 2.412 & 2.301 & 0.140 & 0.218 & 2.392 & 2.321 & 0.132 & 0.211 & 2.355 \\
\hline & & 0.514 & 0.042 & 0.062 & 0.641 & 0.505 & 0.041 & 0.059 & 0.613 & 0.428 & 0.032 & 0.042 & 0.517 \\
\hline & \multirow{2}{*}{$(15,25,30)$} & 2.305 & 0.150 & 0.229 & 2.400 & 2.299 & 0.140 & 0.215 & 2.366 & 2.309 & 0.132 & 0.213 & 2.341 \\
\hline & & 0.485 & 0.033 & 0.057 & 0.632 & 0.481 & 0.035 & 0.056 & 0.629 & 0.376 & 0.028 & 0.029 & 0.488 \\
\hline & \multirow{2}{*}{$(20,25,30)$} & 2.317 & 0.147 & 0.224 & 2.389 & 2.294 & 0.136 & 0.209 & 2.359 & 2.290 & 0.124 & 0.208 & 2.337 \\
\hline & & 0.479 & 0.029 & 0.049 & 0.628 & 0.478 & 0.029 & 0.050 & 0.624 & 0.371 & 0.024 & 0.024 & 0.480 \\
\hline & \multirow{2}{*}{$(20,30,50)$} & 2.301 & 0.132 & 0.201 & 2.340 & 2.290 & 0.129 & 0.208 & 2.338 & 2.287 & 0.113 & 0.207 & 2.324 \\
\hline & & 0.401 & 0.025 & 0.022 & 0.589 & 0.422 & 0.019 & 0.019 & 0.582 & 0.342 & 0.014 & 0.017 & 0.402 \\
\hline & \multirow{2}{*}{$(20,40,50)$} & 2.303 & 0.125 & 0.212 & 2.338 & 2.289 & 0.124 & 0.207 & 2.334 & 2.279 & 0.112 & 0.206 & 2.319 \\
\hline & & 0.398 & 0.019 & 0.021 & 0.560 & 0.410 & 0.018 & 0.018 & 0.563 & 0.332 & 0.012 & 0.013 & 0.388 \\
\hline & \multirow{2}{*}{$(30,40,50)$} & 2.298 & 0.124 & 0.207 & 2.327 & 2.279 & 0.118 & 0.208 & 2.332 & 2.269 & 0.109 & 0.206 & 2.314 \\
\hline & & 0.379 & 0.017 & 0.018 & 0.549 & 0.390 & 0.016 & 0.018 & 0.544 & 0.314 & 0.011 & 0.013 & 0.371 \\
\hline
\end{tabular}

TABle 3: The AV and MSE of parameters estimates for $\Theta(2,0.1,0.2,2.0)$.

\begin{tabular}{|c|c|c|c|c|c|c|c|c|c|c|c|c|c|}
\hline \multirow{2}{*}{$(\eta, \tau)$} & \multirow{2}{*}{$(r, m, n)$} & \multicolumn{4}{|c|}{ MLE } & \multicolumn{4}{|c|}{ MCMC/Prior 0} & \multicolumn{4}{|c|}{ MCMC/Prior 1} \\
\hline & & $\alpha$ & $\beta_{1}$ & $\beta_{1}$ & $\lambda$ & $\alpha$ & $\beta_{1}$ & $\beta_{1}$ & $\lambda$ & $\alpha$ & $\beta_{1}$ & $\beta_{1}$ & $\lambda$ \\
\hline \multirow{12}{*}{$(1.0,1.0)$} & \multirow{2}{*}{$(15,20,30)$} & 2.311 & 0.140 & 0.222 & 2.401 & 2.289 & 0.129 & 0.209 & 2.380 & 2.313 & 0.121 & 0.201 & 2.344 \\
\hline & & 0.490 & 0.032 & 0.051 & 0.620 & 0.490 & 0.031 & 0.048 & 0.594 & 0.412 & 0.022 & 0.031 & 0.500 \\
\hline & \multirow{2}{*}{$(15,25,30)$} & 2.294 & 0.139 & 0.218 & 2.391 & 2.288 & 0.129 & 0.204 & 2.357 & 2.298 & 0.121 & 0.201 & 2.330 \\
\hline & & 0.461 & 0.023 & 0.045 & 0.612 & 0.464 & 0.025 & 0.047 & 0.608 & 0.355 & 0.018 & 0.019 & 0.469 \\
\hline & \multirow{2}{*}{$(20,25,30)$} & 2.307 & 0.138 & 0.215 & 2.379 & 2.285 & 0.126 & 0.200 & 2.348 & 2.288 & 0.114 & 0.200 & 2.329 \\
\hline & & 0.460 & 0.018 & 0.038 & 0.609 & 0.456 & 0.018 & 0.041 & 0.601 & 0.352 & 0.014 & 0.013 & 0.461 \\
\hline & \multirow{2}{*}{$(20,30,50)$} & 2.291 & 0.122 & 0.195 & 2.330 & 2.280 & 0.119 & 0.190 & 2.328 & 2.275 & 0.102 & 0.198 & 2.313 \\
\hline & & 0.385 & 0.014 & 0.011 & 0.574 & 0.403 & 0.008 & 0.009 & 0.564 & 0.325 & 0.005 & 0.008 & 0.385 \\
\hline & \multirow{2}{*}{$(20,40,50)$} & 2.295 & 0.115 & 0.203 & 2.327 & 2.278 & 0.113 & 0.198 & 2.324 & 2.268 & 0.100 & 0.195 & 2.308 \\
\hline & & 0.378 & 0.010 & 0.011 & 0.542 & 0.401 & 0.009 & 0.009 & 0.544 & 0.315 & 0.004 & 0.007 & 0.368 \\
\hline & \multirow{2}{*}{$(30,40,50)$} & 2.288 & 0.112 & 0.195 & 2.318 & 2.268 & 0.109 & 0.200 & 2.322 & 2.249 & 0.100 & 0.197 & 2.301 \\
\hline & & 0.358 & 0.007 & 0.008 & 0.530 & 0.371 & 0.009 & 0.007 & 0.521 & 0.290 & 0.004 & 0.004 & 0.356 \\
\hline
\end{tabular}

TABle 4: The AV and MSE of parameters estimates for $\Theta(2,0.1,0.2,2.0)$.

\begin{tabular}{|c|c|c|c|c|c|c|c|c|c|c|c|c|c|}
\hline \multirow{2}{*}{$(\eta, \tau)$} & \multirow{2}{*}{$(r, m, n)$} & \multicolumn{4}{|c|}{ MLE } & \multicolumn{4}{|c|}{ MCMC/Prior 0} & \multicolumn{4}{|c|}{ MCMC/Prior 1} \\
\hline & & $\alpha$ & $\beta_{1}$ & $\beta_{1}$ & $\lambda$ & $\alpha$ & $\beta_{1}$ & $\beta_{1}$ & $\lambda$ & $\alpha$ & $\beta_{1}$ & $\beta_{1}$ & $\lambda$ \\
\hline \multirow{12}{*}{$(1.2,1.0)$} & \multirow{2}{*}{$(15,20,30)$} & 2.341 & 0.162 & 0.252 & 2.430 & 2.322 & 0.160 & 0.239 & 2.412 & 2.342 & 0.153 & 0.231 & 2.374 \\
\hline & & 0.545 & 0.073 & 0.095 & 0.671 & 0.535 & 0.074 & 0.090 & 0.645 & 0.460 & 0.061 & 0.071 & 0.550 \\
\hline & \multirow{2}{*}{$(15,25,30)$} & 2.325 & 0.170 & 0.249 & 2.419 & 2.318 & 0.161 & 0.236 & 2.386 & 2.328 & 0.151 & 0.233 & 2.360 \\
\hline & & 0.515 & 0.073 & 0.088 & 0.672 & 0.507 & 0.065 & 0.086 & 0.661 & 0.399 & 0.059 & 0.061 & 0.510 \\
\hline & \multirow{2}{*}{$(20,25,30)$} & 2.337 & 0.165 & 0.244 & 2.410 & 2.310 & 0.156 & 0.228 & 2.379 & 2.310 & 0.143 & 0.227 & 2.358 \\
\hline & & 0.509 & 0.060 & 0.081 & 0.660 & 0.508 & 0.058 & 0.081 & 0.658 & 0.399 & 0.055 & 0.056 & 0.503 \\
\hline & \multirow{2}{*}{$(20,30,50)$} & 2.319 & 0.150 & 0.224 & 2.365 & 2.310 & 0.148 & 0.228 & 2.355 & 2.305 & 0.133 & 0.227 & 2.345 \\
\hline & & 0.431 & 0.055 & 0.052 & 0.610 & 0.452 & 0.050 & 0.049 & 0.605 & 0.372 & 0.044 & 0.048 & 0.437 \\
\hline & \multirow{2}{*}{$(20,40,50)$} & 2.323 & 0.145 & 0.233 & 2.358 & 2.307 & 0.144 & 0.226 & 2.355 & 2.299 & 0.131 & 0.227 & 2.338 \\
\hline & & 0.428 & 0.050 & 0.052 & 0.594 & 0.440 & 0.038 & 0.039 & 0.594 & 0.365 & 0.041 & 0.042 & 0.411 \\
\hline & \multirow{2}{*}{$(30,40,50)$} & 2.308 & 0.141 & 0.227 & 2.347 & 2.298 & 0.138 & 0.229 & 2.351 & 2.290 & 0.131 & 0.228 & 2.333 \\
\hline & & 0.408 & 0.047 & 0.048 & 0.578 & 0.421 & 0.047 & 0.048 & 0.574 & 0.345 & 0.042 & 0.045 & 0.399 \\
\hline
\end{tabular}

\section{Monte Carlo Simulations}

Different theoretical results that were developed in this study are assessed and compared in this section through the Monte Carlo study. The numerical computation is formulated to measure the effect of changing the total size $n$ and the sample size $m$ as well as stress time $\eta$ and censoring time $\tau$ and the effect of parameters change. Different three cases of two time $(\tau<\eta, \tau=\eta, \tau>\eta)$ and two values of parameters vector $\Theta=\left(\alpha, \beta_{1}, \beta_{2}, \lambda\right)=\{(2.0,0.1,0.2,2.0),(0.7,1.5,2.0,2.5)\}$ are assumed. Point and interval estimation results are measured. Average (AV) and mean squared error (MSE) are used to 
TABle 5: The AL and CP of parameters estimates for $\Theta(2,0.1,0.2,2.0)$.

\begin{tabular}{|c|c|c|c|c|c|c|c|c|c|c|c|c|c|}
\hline \multirow{2}{*}{$(\eta, \tau)$} & \multirow{2}{*}{$(r, m, n)$} & \multicolumn{4}{|c|}{ MLE } & \multicolumn{4}{|c|}{ MCMC/Prior 0} & \multicolumn{4}{|c|}{ MCMC/Prior 1} \\
\hline & & $\alpha$ & $\beta_{1}$ & $\beta_{1}$ & $\lambda$ & $\alpha$ & $\beta_{1}$ & $\beta_{1}$ & $\lambda$ & $\alpha$ & $\beta_{1}$ & $\beta_{1}$ & $\lambda$ \\
\hline \multirow{12}{*}{$(0.8,1.0)$} & \multirow{2}{*}{$(15,20,30)$} & 4.321 & 0.321 & 0.421 & 5.212 & 4.314 & 0.318 & 0.419 & 5.209 & 4.045 & 0.298 & 0.318 & 5.009 \\
\hline & & 0.89 & 0.90 & 0.90 & 0.88 & 0.90 & 0.91 & 0.91 & 0.90 & 0.90 & 0.92 & 0.90 & 0.91 \\
\hline & \multirow{2}{*}{$(15,25,30)$} & 4.300 & 0.299 & 0.401 & 5.180 & 4.280 & 0.295 & 0.400 & 5.189 & 4.045 & 0.266 & 0.300 & 4.975 \\
\hline & & 0.90 & 0.91 & 0.91 & 0.90 & 0.91 & 0.91 & 0.92 & 0.91 & 0.93 & 0.94 & 0.91 & 0.92 \\
\hline & \multirow{2}{*}{$(20,25,30)$} & 4.271 & 0.268 & 0.375 & 5.154 & 4.255 & 0.265 & 0.381 & 5.174 & 4.018 & 0.239 & 0.271 & 4.944 \\
\hline & & 0.91 & 0.91 & 0.92 & 0.91 & 0.91 & 0.93 & 0.92 & 0.93 & 0.93 & 0.94 & 0.96 & 0.93 \\
\hline & \multirow{2}{*}{$(20,30,50)$} & 4.248 & 0.241 & 0.348 & 5.125 & 4.230 & 0.235 & 0.351 & 5.144 & 4.001 & 0.211 & 0.242 & 4.914 \\
\hline & & 0.92 & 0.92 & 0.92 & 0.90 & 0.93 & 0.93 & 0.93 & 0.93 & 0.93 & 0.95 & 0.95 & 0.92 \\
\hline & \multirow{2}{*}{$(20,40,50)$} & 4.219 & 0.215 & 0.321 & 5.100 & 4.201 & 0.203 & 0.318 & 5.115 & 3.971 & 0.180 & 0.215 & 4.850 \\
\hline & & 0.91 & 0.93 & 0.93 & 0.91 & 0.93 & 0.94 & 0.93 & 0.93 & 0.94 & 0.97 & 0.95 & 0.93 \\
\hline & \multirow{2}{*}{$(30,40,50)$} & 4.185 & 0.194 & 0.300 & 4.990 & 4.150 & 0.164 & 0.270 & 5.070 & 3.941 & 0.149 & 0.176 & 4.817 \\
\hline & & 0.92 & 0.93 & 0.93 & 0.92 & 0.93 & 0.94 & 0.93 & 0.93 & 0.94 & 0.93 & 0.95 & 0.94 \\
\hline
\end{tabular}

TABLE 6: The AL and CP of parameters estimates for $\Theta(2,0.1,0.2,2.0)$.

\begin{tabular}{|c|c|c|c|c|c|c|c|c|c|c|c|c|c|}
\hline \multirow{2}{*}{$(\eta, \tau)$} & \multirow{2}{*}{$(r, m, n)$} & \multicolumn{4}{|c|}{ MLE } & \multicolumn{4}{|c|}{ MCMC/Prior 0} & \multicolumn{4}{|c|}{ MCMC/Prior 1} \\
\hline & & $\alpha$ & $\beta_{1}$ & $\beta_{1}$ & $\lambda$ & $\alpha$ & $\beta_{1}$ & $\beta_{1}$ & $\lambda$ & $\alpha$ & $\beta_{1}$ & $\beta_{1}$ & $\lambda$ \\
\hline \multirow{12}{*}{$(1.0,1.0)$} & \multirow{2}{*}{$(15,20,30)$} & 4.285 & 0.291 & 0.387 & 5.088 & 4.300 & 0.290 & 0.400 & 5.187 & 4.019 & 0.270 & 0.294 & 4.980 \\
\hline & & 0.90 & 0.91 & 0.90 & 0.89 & 0.91 & 0.91 & 0.91 & 0.92 & 0.90 & 0.92 & 0.91 & 0.91 \\
\hline & \multirow{2}{*}{$(15,25,30)$} & 4.272 & 0.271 & 0.375 & 5.152 & 4.253 & 0.269 & 0.374 & 5.158 & 4.014 & 0.249 & 0.274 & 4.948 \\
\hline & & 0.91 & 0.91 & 0.92 & 0.90 & 0.91 & 0.91 & 0.92 & 0.93 & 0.93 & 0.94 & 0.91 & 0.94 \\
\hline & \multirow{2}{*}{$(20,25,30)$} & 4.242 & 0.235 & 0.342 & 5.118 & 4.255 & 0.239 & 0.351 & 5.149 & 3.980 & 0.208 & 0.241 & 4.915 \\
\hline & & 0.92 & 0.93 & 0.92 & 0.92 & 0.91 & 0.93 & 0.92 & 0.95 & 0.93 & 0.94 & 0.96 & 0.94 \\
\hline & \multirow{2}{*}{$(20,30,50)$} & 4.219 & 0.212 & 0.318 & 5.100 & 4.204 & 0.208 & 0.328 & 5.113 & 3.971 & 0.185 & 0.214 & 4.875 \\
\hline & & 0.93 & 0.92 & 0.94 & 0.90 & 0.93 & 0.93 & 0.97 & 0.93 & 0.93 & 0.95 & 0.97 & 0.92 \\
\hline & \multirow{2}{*}{$(20,40,50)$} & 4.181 & 0.185 & 0.284 & 5.079 & 4.172 & 0.169 & 0.280 & 5.081 & 3.940 & 0.151 & 0.179 & 4.821 \\
\hline & & 0.96 & 0.92 & 0.93 & 0.91 & 0.93 & 0.93 & 0.93 & 0.93 & 0.94 & 0.97 & 0.95 & 0.94 \\
\hline & \multirow{2}{*}{$(30,40,50)$} & 4.158 & 0.166 & 0.274 & 4.965 & 4.118 & 0.135 & 0.241 & 5.040 & 3.915 & 0.118 & 0.148 & 4.782 \\
\hline & & 0.92 & 0.94 & 0.93 & 0.92 & 0.93 & 0.94 & 0.92 & 0.93 & 0.94 & 0.95 & 0.95 & 0.94 \\
\hline
\end{tabular}

TABle 7: The AL and CP of parameters estimates for $\Theta(2,0.1,0.2,2.0)$.

\begin{tabular}{|c|c|c|c|c|c|c|c|c|c|c|c|c|c|}
\hline \multirow{2}{*}{$(\eta, \tau)$} & \multirow{2}{*}{$(r, m, n)$} & \multicolumn{4}{|c|}{ MLE } & \multicolumn{4}{|c|}{ MCMC/Prior 0} & \multicolumn{4}{|c|}{ MCMC/Prior 1} \\
\hline & & $\alpha$ & $\beta_{1}$ & $\beta_{1}$ & $\lambda$ & $\alpha$ & $\beta_{1}$ & $\beta_{1}$ & $\lambda$ & $\alpha$ & $\beta_{1}$ & $\beta_{1}$ & $\lambda$ \\
\hline \multirow{12}{*}{$(1.2,1.0)$} & \multirow{2}{*}{$(15,20,30)$} & 4.371 & 0.369 & 0.470 & 5.269 & 4.370 & 0.371 & 0.466 & 5.255 & 4.098 & 0.345 & 0.361 & 5.057 \\
\hline & & 0.88 & 0.89 & 0.90 & 0.88 & 0.91 & 0.90 & 0.91 & 0.91 & 0.91 & 0.92 & 0.90 & 0.91 \\
\hline & \multirow{2}{*}{$(15,25,30)$} & 4.347 & 0.245 & 0.453 & 5.230 & 4.330 & 0.345 & 0.450 & 5.238 & 4.091 & 0.299 & 0.348 & 5001 \\
\hline & & 0.89 & 0.90 & 0.91 & 0.91 & 0.91 & 0.92 & 0.92 & 0.93 & 0.93 & 0.94 & 0.93 & 0.92 \\
\hline & \multirow{2}{*}{$(20,25,30)$} & 4.318 & 0.310 & 0.415 & 5.198 & 4.294 & 0.310 & 0.428 & 5.221 & 4.071 & 0.288 & 0.321 & 4.989 \\
\hline & & 0.90 & 0.91 & 0.92 & 0.90 & 0.92 & 0.93 & 0.92 & 0.93 & 0.94 & 0.94 & 0.96 & 0.97 \\
\hline & \multirow{2}{*}{$(20,30,50)$} & 4.291 & 0.287 & 0.389 & 5.176 & 4.280 & 0.279 & 0.398 & 5.188 & 4.056 & 0.261 & 0.289 & 4.961 \\
\hline & & 0.92 & 0.91 & 0.92 & 0.90 & 0.92 & 0.93 & 0.93 & 0.93 & 0.93 & 0.93 & 0.95 & 0.94 \\
\hline & \multirow{2}{*}{$(20,40,50)$} & 4.270 & 0.265 & 0.372 & 5.164 & 4.245 & 0.253 & 0.359 & 5.164 & 4.008 & 0.220 & 0.266 & 4.894 \\
\hline & & 0.92 & 0.93 & 0.90 & 0.91 & 0.93 & 0.94 & 0.93 & 0.92 & 0.94 & 0.97 & 0.94 & 0.93 \\
\hline & \multirow{2}{*}{$(30,40,50)$} & 4.221 & 0.243 & 0.351 & 5.011 & 4.194 & 0.210 & 0.320 & 5.123 & 3.990 & 0.187 & 0.516 & 4.866 \\
\hline & & 0.92 & 0.90 & 0.93 & 0.91 & 0.93 & 0.94 & 0.93 & 0.93 & 0.94 & 0.94 & 0.95 & 0.96 \\
\hline
\end{tabular}

measure point estimate and average lengths (AL) and the probability coverage (CP) are used to measure interval estimate. For Bayes estimation with MCMC methods, prior parameters are selected to satisfy the expectation of gamma prior as $E\left(\Theta_{l}\right)=\left(a_{l} / b_{l}\right) \cong \Theta_{l}, l=1,2,3$. Then, informative prior information (Prior 1) and noninformative prior are obtained when the posterior distribution is proportional with the likelihood function (Prior 0). Also, Chan is built for 11,000 iterations with the first 1000 as bur-in. Average Bayes estimates, mean squared errors (MSEs), coverage 
TABLE 8: The AV and MSE of parameters estimates for $\Theta(1.0,0.8,1.0,2.5)$.

\begin{tabular}{|c|c|c|c|c|c|c|c|c|c|c|c|c|c|}
\hline \multirow{2}{*}{$(\eta, \tau)$} & \multirow{2}{*}{$(r, m, n)$} & \multicolumn{4}{|c|}{ MLE } & \multicolumn{4}{|c|}{ MCMC/Prior 0} & \multicolumn{4}{|c|}{ MCMC/Prior 1} \\
\hline & & $\alpha$ & $\beta_{1}$ & $\beta_{1}$ & $\lambda$ & $\alpha$ & $\beta_{1}$ & $\beta_{1}$ & $\lambda$ & $\alpha$ & $\beta_{1}$ & $\beta_{1}$ & $\lambda$ \\
\hline \multirow{12}{*}{$(0.3,0.5)$} & \multirow{2}{*}{$(15,20,30)$} & 1.235 & 1.001 & 1.321 & 2.821 & 1.214 & 0.982 & 1.301 & 2.805 & 1.201 & 0.854 & 1.241 & 2.754 \\
\hline & & 0.254 & 0.221 & 0.243 & 0.647 & 0.241 & 0.213 & 0.232 & 0.641 & 0.185 & 0.167 & 0.200 & 0.425 \\
\hline & \multirow{2}{*}{$(15,25,30)$} & 1.219 & 0.985 & 1.307 & 2.809 & 1.200 & 0.968 & 1.280 & 2.791 & 1.189 & 0.842 & 1.229 & 2.742 \\
\hline & & 0.237 & 0.205 & 0.218 & 0.622 & 0.221 & 0.190 & 0.214 & 0.628 & 0.165 & 0.155 & 0.193 & 0.417 \\
\hline & \multirow{2}{*}{$(20,25,30)$} & 1.221 & 0.982 & 1.313 & 2.814 & 1.201 & 0.955 & 1.275 & 2.792 & 1.178 & 0.840 & 1.214 & 2.735 \\
\hline & & 0.227 & 0.201 & 0.212 & 0.619 & 0.218 & 0.183 & 0.208 & 0.614 & 0.145 & 0.147 & 0.190 & 0.409 \\
\hline & \multirow{2}{*}{$(20,30,50)$} & 1.214 & 0.977 & 1.307 & 2.817 & 1.194 & 0.948 & 1.269 & 2.790 & 1.160 & 0.825 & 1.204 & 2.727 \\
\hline & & 0.209 & 0.192 & 0.200 & 0.604 & 0.201 & 0.164 & 0.201 & 0.601 & 0.139 & 0.132 & 0.179 & 0.401 \\
\hline & \multirow{2}{*}{$(20,40,50)$} & 1.195 & 0.899 & 1.265 & 2.800 & 1.191 & 0.888 & 1.255 & 2.718 & 1.124 & 0.801 & 1.176 & 2.702 \\
\hline & & 0.182 & 0.176 & 0.166 & 0.550 & 0.183 & 0.161 & 0.164 & 0.547 & 0.101 & 0.108 & 0.151 & 0.382 \\
\hline & \multirow{2}{*}{$(30,40,50)$} & 1.184 & 0.875 & 1.240 & 2.741 & 1.179 & 0.872 & 1.221 & 2.719 & 1.127 & 0.789 & 1.154 & 2.701 \\
\hline & & 0.174 & 0.160 & 0.142 & 0.514 & 0.156 & 0.140 & 0.133 & 0.519 & 0.099 & 0.089 & 0.132 & 0.314 \\
\hline
\end{tabular}

TABle 9: The AV and MSE of parameters estimates for $\Theta(1.0,0.8,1.0,2.5)$.

\begin{tabular}{|c|c|c|c|c|c|c|c|c|c|c|c|c|c|}
\hline \multirow{2}{*}{$(\eta, \tau)$} & \multirow{2}{*}{$(r, m, n)$} & \multicolumn{4}{|c|}{ MLE } & \multicolumn{4}{|c|}{ MCMC/Prior 0} & \multicolumn{4}{|c|}{ MCMC/Prior 1} \\
\hline & & $\alpha$ & $\beta_{1}$ & $\beta_{1}$ & $\lambda$ & $\alpha$ & $\beta_{1}$ & $\beta_{1}$ & $\lambda$ & $\alpha$ & $\beta_{1}$ & $\beta_{1}$ & $\lambda$ \\
\hline \multirow{12}{*}{$(0.5,0.5)$} & \multirow{2}{*}{$(15,20,30)$} & 1.219 & 0945 & 1.300 & 2.800 & 1.184 & 0.973 & 1.290 & 2.780 & 1.212 & 0.866 & 1.254 & 2.767 \\
\hline & & 0.233 & 0.207 & 0.219 & 0.618 & 0.215 & 0.200 & 0.211 & 0.619 & 0.155 & 0.149 & 0.179 & 0.403 \\
\hline & \multirow{2}{*}{$(15,25,30)$} & 1.201 & 0.971 & 1.219 & 2.800 & 1.203 & 0.954 & 1.275 & 2.787 & 1.180 & 0.845 & 1.217 & 2.732 \\
\hline & & 0.219 & 0.187 & 0.204 & 0.604 & 0.200 & 0.172 & 0.189 & 0.611 & 0.152 & 0.141 & 0.173 & 0.401 \\
\hline & \multirow{2}{*}{$(20,25,30)$} & 1.211 & 0.975 & 1.300 & 2.801 & 1.192 & 0.947 & 1.269 & 2.790 & 1.179 & 0.842 & 1.207 & 2.725 \\
\hline & & 0.209 & 0.175 & 0.200 & 0.601 & 0.197 & 0.164 & 0.187 & 0.600 & 0.132 & 0.125 & 0.174 & 0.390 \\
\hline & \multirow{2}{*}{$(20,30,50)$} & 1.204 & 0.961 & 1.310 & 2.807 & 1.182 & 0.941 & 1.260 & 2.784 & 1.163 & 0.817 & 1.191 & 2.708 \\
\hline & & 0.191 & 0.179 & 0.183 & 0.581 & 0.180 & 0.162 & 0.189 & 0.580 & 0.117 & 0.111 & 0.160 & 0.382 \\
\hline & \multirow{2}{*}{$(20,40,50)$} & 1.190 & 0.880 & 1.260 & 2.803 & 1.190 & 0.878 & 1.247 & 2.725 & 1.132 & 0.715 & 1.160 & 2.650 \\
\hline & & 0.161 & 0.156 & 0.149 & 0.535 & 0.168 & 0.145 & 0.145 & 0.538 & 0.089 & 0.079 & 0.138 & 0.365 \\
\hline & \multirow{2}{*}{$(30,40,50)$} & 1.176 & 0.871 & 1.233 & 2.742 & 1.165 & 0.869 & 1.217 & 2.731 & 1.130 & 0.776 & 1.151 & 2.690 \\
\hline & & 0.155 & 0.143 & 0.128 & 0.500 & 0.141 & 0.128 & 0.117 & 0.504 & 0.081 & 0.076 & 0.114 & 0.301 \\
\hline
\end{tabular}

TABle 10: The AV and MSE of parameters estimates for $\Theta(1.0,0.8,1.0,2.5)$.

\begin{tabular}{|c|c|c|c|c|c|c|c|c|c|c|c|c|c|}
\hline \multirow{2}{*}{$(\eta, \tau)$} & \multirow{2}{*}{$(r, m, n)$} & \multicolumn{4}{|c|}{ MLE } & \multicolumn{4}{|c|}{ MCMC/Prior 0} & \multicolumn{4}{|c|}{ MCMC/Prior 1} \\
\hline & & $\alpha$ & $\beta_{1}$ & $\beta_{1}$ & $\lambda$ & $\alpha$ & $\beta_{1}$ & $\beta_{1}$ & $\lambda$ & $\alpha$ & $\beta_{1}$ & $\beta_{1}$ & $\lambda$ \\
\hline \multirow{12}{*}{$(0.3,0.5)$} & \multirow{2}{*}{$(15,20,30)$} & 1.235 & 1.001 & 1.321 & 2.821 & 1.214 & 0.982 & 1.301 & 2.805 & 1.201 & 0.854 & 1.241 & 2.754 \\
\hline & & 0.254 & 0.221 & 0.243 & 0.647 & 0.241 & 0.213 & 0.232 & 0.641 & 0.185 & 0.167 & 0.200 & 0.425 \\
\hline & \multirow{2}{*}{$(15,25,30)$} & 1.219 & 0.985 & 1.307 & 2.809 & 1.200 & 0.968 & 1.280 & 2.791 & 1.189 & 0.842 & 1.229 & 2.742 \\
\hline & & 0.237 & 0.205 & 0.218 & 0.622 & 0.221 & 0.190 & 0.214 & 0.628 & 0.165 & 0.155 & 0.193 & 0.417 \\
\hline & \multirow{2}{*}{$(20,25,30)$} & 1.221 & 0.982 & 1.313 & 2.814 & 1.201 & 0.955 & 1.275 & 2.792 & 1.178 & 0.840 & 1.214 & 2.735 \\
\hline & & 0.227 & 0.201 & 0.212 & 0.619 & 0.218 & 0.183 & 0.208 & 0.614 & 0.145 & 0.147 & 0.190 & 0.409 \\
\hline & \multirow{2}{*}{$(20,30,50)$} & 1.214 & 0.977 & 1.307 & 2.817 & 1.194 & 0.948 & 1.269 & 2.790 & 1.160 & 0.825 & 1.204 & 2.727 \\
\hline & & 0.209 & 0.192 & 0.200 & 0.604 & 0.201 & 0.164 & 0.201 & 0.601 & 0.139 & 0.132 & 0.179 & 0.401 \\
\hline & \multirow{2}{*}{$(20,40,50)$} & 1.195 & 0.899 & 1.265 & 2.800 & 1.191 & 0.888 & 1.255 & 2.718 & 1.124 & 0.801 & 1.176 & 2.702 \\
\hline & & 0.182 & 0.176 & 0.166 & 0.550 & 0.183 & 0.161 & 0.164 & 0.547 & 0.101 & 0.108 & 0.151 & 0.382 \\
\hline & \multirow{2}{*}{$(30,40,50)$} & 1.184 & 0.875 & 1.240 & 2.741 & 1.179 & 0.872 & 1.221 & 2.719 & 1.127 & 0.789 & 1.154 & 2.701 \\
\hline & & 0.174 & 0.160 & 0.142 & 0.514 & 0.156 & 0.140 & 0.133 & 0.519 & 0.099 & 0.089 & 0.132 & 0.314 \\
\hline
\end{tabular}


TABle 11: The AL and CP of parameters estimates for $\Theta(1.0,0.8,1.0,2.5)$.

\begin{tabular}{|c|c|c|c|c|c|c|c|c|c|c|c|c|c|}
\hline \multirow{2}{*}{$(\eta, \tau)$} & \multirow{2}{*}{$(r, m, n)$} & \multicolumn{4}{|c|}{ MLE } & \multicolumn{4}{|c|}{ MCMC/Prior 0} & \multicolumn{4}{|c|}{ MCMC/Prior 1} \\
\hline & & $\alpha$ & $\beta_{1}$ & $\beta_{1}$ & $\lambda$ & $\alpha$ & $\beta_{1}$ & $\beta_{1}$ & $\lambda$ & $\alpha$ & $\beta_{1}$ & $\beta_{1}$ & $\lambda$ \\
\hline \multirow{12}{*}{$(0.3,0.5)$} & \multirow{2}{*}{$(15,20,30)$} & 2.824 & 2.541 & 3.104 & 5.512 & 2.801 & 2.549 & 3.097 & 5.489 & 2.612 & 2.325 & 2.900 & 5.342 \\
\hline & & 0.89 & 0.88 & 0.89 & 0.87 & 0.89 & 0.90 & 0.90 & 0.88 & 0.90 & 0.90 & 0.90 & 0.90 \\
\hline & \multirow{2}{*}{$(15,25,30)$} & 2.804 & 2.525 & 3.077 & 5.500 & 2.785 & 2.525 & 3.077 & 5.468 & 2.591 & 2.301 & 2.879 & 5.324 \\
\hline & & 0.90 & 0.90 & 0.90 & 0.90 & 0.91 & 0.91 & 0.91 & 0.91 & 0.91 & 0.92 & 0.91 & 0.92 \\
\hline & \multirow{2}{*}{$(20,25,30)$} & 2.781 & 2.507 & 3.059 & 5.480 & 2.766 & 2.508 & 3.059 & 5.455 & 2.579 & 2.284 & 2.862 & 5.304 \\
\hline & & 0.91 & 0.91 & 0.92 & 0.92 & 0.91 & 0.93 & 0.92 & 0.92 & 0.93 & 0.94 & 0.98 & 0.93 \\
\hline & \multirow{2}{*}{$(20,30,50)$} & 2.759 & 2.487 & 3.042 & 5.461 & 2.745 & 2.592 & 3.040 & 5.439 & 2.565 & 2.271 & 2.842 & 5.281 \\
\hline & & 0.91 & 0.92 & 0.91 & 0.90 & 0.93 & 0.93 & 0.91 & 0.93 & 0.93 & 0.92 & 0.92 & 0.92 \\
\hline & \multirow{2}{*}{$(20,40,50)$} & 2.744 & 2.464 & 3.031 & 5.449 & 2.736 & 2.581 & 3.024 & 5.418 & 2.548 & 2.255 & 2.819 & 5.260 \\
\hline & & 0.912 & 0.93 & 0.92 & 0.90 & 0.93 & 0.94 & 0.93 & 0.93 & 0.93 & 0.92 & 0.94 & 0.93 \\
\hline & \multirow{2}{*}{$(30,40,50)$} & 2.719 & 2.439 & 3.008 & 5.413 & 2.714 & 2.547 & 3.004 & 5.400 & 2.521 & 2.228 & 2.801 & 5.239 \\
\hline & & 0.92 & 0.92 & 0.93 & 0.92 & 0.92 & 0.94 & 0.93 & 0.94 & 0.94 & 0.93 & 0.95 & 0.95 \\
\hline
\end{tabular}

TABle 12: The AL and CP of parameters estimates for $\Theta(1.0,0.8,1.0,2.5)$.

\begin{tabular}{|c|c|c|c|c|c|c|c|c|c|c|c|c|c|}
\hline \multirow{2}{*}{$(\eta, \tau)$} & \multirow{2}{*}{$(r, m, n)$} & \multicolumn{4}{|c|}{ MLE } & \multicolumn{4}{|c|}{ MCMC/Prior 0} & \multicolumn{4}{|c|}{ MCMC/Prior 1} \\
\hline & & $\alpha$ & $\beta_{1}$ & $\beta_{1}$ & $\lambda$ & $\alpha$ & $\beta_{1}$ & $\beta_{1}$ & $\lambda$ & $\alpha$ & $\beta_{1}$ & $\beta_{1}$ & $\lambda$ \\
\hline \multirow{12}{*}{$(0.5,0.5)$} & \multirow{2}{*}{$(15,20,30)$} & 2.801 & 2.514 & 3.076 & 5.500 & 2.783 & 2.515 & 3.072 & 5.471 & 2.593 & 2.304 & 2.879 & 5.319 \\
\hline & & 0.90 & 0.90 & 0.89 & 0.89 & 0.90 & 0.90 & 0.91 & 0.90 & 0.90 & 0.91 & 0.90 & 0.92 \\
\hline & \multirow{2}{*}{$(15,25,30)$} & 2.782 & 2.507 & 3.058 & 5.483 & 2.769 & 2.507 & 3.061 & 5.454 & 2.576 & 2.279 & 2.864 & 5.308 \\
\hline & & 0.92 & 0.91 & 0.90 & 0.91 & 0.92 & 0.91 & 0.92 & 0.91 & 0.91 & 0.92 & 0.93 & 0.93 \\
\hline & \multirow{2}{*}{$(20,25,30)$} & 2.756 & 2.486 & 3.035 & 5.464 & 2.748 & 2.492 & 3.041 & 5.441 & 2.568 & 2.269 & 2.847 & 5.292 \\
\hline & & 0.92 & 0.93 & 0.92 & 0.93 & 0.91 & 0.93 & 0.94 & 0.92 & 0.93 & 0.95 & 0.96 & 0.95 \\
\hline & \multirow{2}{*}{$(20,30,50)$} & 2.743 & 2.461 & 3.020 & 5.443 & 2.738 & 2.582 & 3.025 & 5.428 & 2.547 & 2.256 & 2.821 & 5.255 \\
\hline & & 0.93 & 0.92 & 0.93 & 0.93 & 0.93 & 0.93 & 0.93 & 0.93 & 0.93 & 0.95 & 0.94 & 0.93 \\
\hline & \multirow{2}{*}{$(20,40,50)$} & 2.725 & 2.451 & 3.014 & 5.432 & 2.718 & 2.563 & 3.011 & 5.408 & 2.531 & 2.241 & 2.808 & 5.245 \\
\hline & & 0.92 & 0.93 & 0.92 & 0.90 & 0.94 & 0.94 & 0.93 & 0.93 & 0.94 & 0.92 & 0.94 & 0.96 \\
\hline & \multirow{2}{*}{$(30,40,50)$} & 2.719 & 2.439 & 3.008 & 5.413 & 2.714 & 2.547 & 3.004 & 5.400 & 2.521 & 2.228 & 2.801 & 5.239 \\
\hline & & 0.92 & 0.92 & 0.93 & 0.92 & 0.92 & 0.94 & 0.93 & 0.94 & 0.94 & 0.93 & 0.95 & 0.95 \\
\hline
\end{tabular}

TABLE 13: The AL and CP of parameters estimates for $\Theta(1.0,0.8,1.0,2.5)$.

\begin{tabular}{|c|c|c|c|c|c|c|c|c|c|c|c|c|c|}
\hline \multirow{2}{*}{$(\eta, \tau)$} & \multirow{2}{*}{$(r, m, n)$} & \multicolumn{4}{|c|}{ MLE } & \multicolumn{4}{|c|}{ MCMC/Prior 0} & \multicolumn{4}{|c|}{ MCMC/Prior 1} \\
\hline & & $\alpha$ & $\beta_{1}$ & $\beta_{1}$ & $\lambda$ & $\alpha$ & $\beta_{1}$ & $\beta_{1}$ & $\lambda$ & $\alpha$ & $\beta_{1}$ & $\beta_{1}$ & $\lambda$ \\
\hline \multirow{12}{*}{$(0.7,0.5)$} & \multirow{2}{*}{$(15,20,30)$} & 2.861 & 2.579 & 3.142 & 5.544 & 2.832 & 2.580 & 3.180 & 5.511 & 2.645 & 2.349 & 2.932 & 5.361 \\
\hline & & 0.89 & 0.88 & 0.89 & 0.88 & 0.91 & 0.90 & 0.91 & 0.89 & 0.90 & 0.90 & 0.90 & 0.91 \\
\hline & \multirow{2}{*}{$(15,25,30)$} & 2.835 & 2.559 & 3.103 & 5.528 & 2.810 & 2.561 & 3.098 & 5.488 & 2.670 & 2.342 & 2.915 & 5.357 \\
\hline & & 0.90 & 0.91 & 0.90 & 0.91 & 0.91 & 0.91 & 0.91 & 0.92 & 0.91 & 0.92 & 0.91 & 0.92 \\
\hline & \multirow{2}{*}{$(20,25,30)$} & 2.799 & 2.538 & 3.071 & 5.499 & 2.784 & 2.541 & 3.075 & 5.478 & 2.611 & 2.315 & 2.881 & 5.338 \\
\hline & & 0.90 & 0.91 & 0.93 & 0.92 & 0.91 & 0.93 & 0.92 & 0.92 & 0.94 & 0.94 & 0.98 & 0.92 \\
\hline & \multirow{2}{*}{$(20,30,50)$} & 2.782 & 2.499 & 3.073 & 5.486 & 2.774 & 2.615 & 3.069 & 5.470 & 2.592 & 2.298 & 2.869 & 5.298 \\
\hline & & 0.92 & 0.90 & 0.91 & 0.91 & 0.93 & 0.93 & 0.91 & 0.94 & 0.93 & 0.92 & 0.93 & 0.92 \\
\hline & \multirow{2}{*}{$(20,40,50)$} & 2.771 & 2.492 & 3.059 & 5.480 & 2.764 & 2.598 & 3.052 & 5.441 & 2.563 & 2.277 & 2.842 & 5.285 \\
\hline & & 0.90 & 0.93 & 0.92 & 0.92 & 0.93 & 0.94 & 0.94 & 0.93 & 0.93 & 0.92 & 0.94 & 0.95 \\
\hline & \multirow{2}{*}{$(30,40,50)$} & 2.741 & 2.458 & 3.032 & 5.439 & 2.742 & 2.568 & 3.032 & 5.429 & 2.544 & 2.253 & 2.834 & 5.257 \\
\hline & & 0.92 & 0.91 & 0.93 & 0.92 & 0.92 & 0.94 & 0.93 & 0.92 & 0.94 & 0.93 & 0.93 & 0.93 \\
\hline
\end{tabular}

percentages, and average confidence interval lengths based on 1000 replications are reported. The results are shown in Tables 2-13.

\section{Conclusions}

A suitable censoring scheme and a suitable accelerated type for competing risk populations are developed. The results are computed when the products have Chen lifetime distribution. The model parameters are estimated with MLEs and Bayes methods; then, the numerical results are reported in Tables 2-13. The following comments are observed for the numerical results.

(1) From all tables, the proposed methods serve well for all choices. 
(2) The numerical results which are obtained from MLEs and Bayes with noninformative prior are approximately similar.

(3) The Bayes method under informative prior serves very well compared to the other cases.

(4) The censoring scheme of type-I GHCS has saved the minimum number of failures which present suitable numerical results of all choices

(5) For the effective $(r, m)$ sample increases, the MSEs and the AL of different estimators are reduced.

(6) The best results are obtained for the cases of $\eta \cong \tau$.

\section{Data Availability}

No data were used to support the findings of this study.

\section{Conflicts of Interest}

The authors have no conflicts of interest regarding the publication of the paper.

\section{Acknowledgments}

This research was funded by the Deanship of Scientific Research, Taif University, KSA (Research Group no. 1- 1441100).

\section{References}

[1] R. D. Gupta and D. Kundu, "Hybrid censoring schemes with exponential failure distribution," Communications in Statistics-Theory and Methods, vol. 27, no. 12, pp. 3065-3083, 1998.

[2] D. Kundu and B. Pradhan, "Estimating the parameters of the generalized exponential distribution in presence of hybrid censoring," Communications in Statistics-Theory and Methods, vol. 38, no. 12, pp. 2030-2041, 2009.

[3] A. Childs, B. Chandrasekar, N. Balakrishnan, and D. Kundu, "Exact likelihood inference based on Type-I and Type-II hybrid censored samples from the exponential distribution," Annals of the Institute of Statistical Mathematics, vol. 55, no. 2, pp. 319-330, 2003.

[4] M. G. M. Ghazal, "Prediction of exponentiated family distributions observables under type-II hybrid censored data," Journal of Statistics Applications \& Probability, vol. 7, no. 2, pp. 307-319, 2018.

[5] B. Chandrasekar, A. Childs, and N. Balakrishnan, "Exact likelihood inference for the exponential distribution under generalized Type-I and Type-II hybrid censoring," Naval Research Logistics, vol. 51, no. 7, pp. 994-1004, 2004.

[6] D. R. Cox, "The analysis of exponentially distributed life-times with two types of failure," Journal of the Royal Statistical Society: Series B (Methodological), vol. 21, no. 2, pp. 411-421, 1959.

[7] H. A. David and M. L. Moeschberger, The Theory of Competing Risks, Grin, London, UK, 1978.

[8] M. J. Crowder, Classical Competing Risks, Chapman \& Hall, London. UK, 2001.

[9] N. Balakrishnan and D. Han, "Exact inference for a simple step-stress model with competing risks for failure from exponential distribution under Type-II censoring," Journal of
Statistical Planning and Inference, vol. 138, no. 12, pp. 4172 4186, 2008.

[10] A. Ganguly and D. Kundu, "Analysis of simple step-stress model in presence of competing risks," Journal of Statistical Computation and Simulation, vol. 86, no. 10, pp. 1989-2006, 2016.

[11] H. H. Abu-Zinadah and N. Sayed-Ahmed, "Competing risks model with partially step-stress accelerate life tests in analyses lifetime chen data under type-II censoring scheme," Open Physics, vol. 17, no. 1, pp. 192-199, 2019.

[12] W. Nelson, Accelerated Testing: Statistical Models, Test Plans and Data Analysis, Wiley, NewYork, NY, USA, 1990.

[13] C. M. Kim and D. S. Bai, "Analyses of accelerated life test data under two failure modes," International Journal of Reliability, Quality and Safety Engineering, vol. 09, no. 02, pp. 111-125, 2002.

[14] V. Bagdonavicius and M. Nikulin, Accelerated Life Models: Modeling and Statistical Analysis, Chapman and Hall/CRC Press, Boca Raton, FL, USA, 2002.

[15] E. K. AL-Hussaini and A. H. Abdel-Hamid, "Bayesian estimation of the parameters, reliability and hazard rate functions of mixtures under accelerated life tests," Communications in Statistics - Simulation and Computation, vol. 33, no. 4, pp. 963-982, 2004.

[16] E. K. AL-Hussaini and A. H. Abdel-Hamid, "Accelerated life tests under finite mixture models," Journal of Statistical Computation and Simulation, vol. 76, no. 8, pp. 673-690, 2006.

[17] D. S. Bai, M. S. Kim, and S. H. Lee, "Optimum simple stepstress accelerated life tests with censoring," IEEE Transactions on Reliability, vol. 38, no. 5, pp. 528-532, 1989.

[18] R. Wang and H. Fei, "Statistical inference of Weibull distribution for tampered failure rate model in progressive stress accelerated life testing," IEEE Transactions on Reliability, vol. 17, pp. 237-243, 2004.

[19] A. H. Abdel-Hamid and E. K. Al-Hussaini, "Progressive stress accelerated life tests under finite mixture models," Metrika, vol. 66, no. 2, pp. 213-231, 2007.

[20] G. K. Bhattacharyya and Z. Soejoeti, "A tampered failure rate model for step-stress accelerated life test," Communications in Statistics-Theory and Methods, vol. 18, no. 5, pp. 1627-1643, 1989.

[21] H. Seunggeun and J. Lee, "Constant-stress partially accelerated life testing for log-logistic distribution with censored data," IEEE Transactions on Reliability, vol. 2, pp. 193-201, 2015.

[22] A. A. Tahani and A. A. Soliman, "Estimating the Pareto parameters under progressive censoring data for constantpartially accelerated life tests," IEEE Transactions on Reliability, vol. 85, pp. 917-934, 2015.

[23] G. A. Abd-Elmougod and E. E. Mahmoud, "Paramters estimation of compound Rayleigh distribution under an adaptive type-II progressively hybrid censored data for constant partially accelerated life tests," Global Journal of Pure and Applied Mathematics, vol. 13, pp. 8361-8372, 2016.

[24] A. A. Soliman, G. A. Abd-Elmougod, and M. M. Al- Sobhi, "Estimation in step-stress partially accelerated life tests for the Chen distribution using progressive Type-II censoring," Applied Mathematics \& Information Sciences, vol. 11, no. 1, pp. 325-332, 2017.

[25] A. M. Almarashi, AlgarniA, G. A. Abd-Elmougod, and S. M. Sayed Abdel-Khalek, "MCMC in estimation for generalized exponential distribution with constant partially 
accelerated life tests under type-II censoring scheme," Transylvanian Review, vol. 32, pp. 8369-8376, 2019.

[26] A. M. Almarashia, A. Ali, G. A. Abd-Elmougod, and S. AbdelKhalek, "Statistical analysis of Rayleigh competing risks models based on partially step stress Type-II censoring samples," Journal of Nonlinear Science and Applications, vol. 12, pp. 230-238, 2019.

[27] Q. Guan and Y. Tang, "Optimal step-stress test under Type-I censoring for multivariate exponential distribution," Journal of Statistical Planning and Inference, vol. 142, no. 7, pp. 1908-1923, 2012. 